\title{
Territori in transizione: nuove reti e nuove identità per le economie e le società locali
}

\author{
ENZO RULLANI
}

\section{Che cosa è, oggi, il territorio?}

Il territorio - che abbiamo conosciuto e frequentato fino a un decennio fa - sta mutando con una rapidità che non ci ha ancora dato il tempo di rivedere le nostre categorie mentali e rappresentazioni con cui gli diamo forma e significato. Cosicchè continuiamo a vedere quello che sta accadendo nei luoghi che abitiamo o in cui lavoriamo con occhi - e presupposti - che non corrispondono più alla realtà emergente dei nostri giorni.

Dunque, che cosa è, oggi, il territorio?

È la domanda che implicitamente percorre tutti i contributi di questo numero di Sinergie e che nel convegno di Lecce è stata implicitamente oggetto di discussione. La sua esplicitazione - cosa che cercherò di fare in poche parole - è la premessa per ordinare le diverse visioni e applicazioni che si rifanno al territorio.

Il territorio oggi entra a far parte della nostra esperienza in tanti modi diversi.

Una volta, quando la vita e il lavoro si svolgevano entro il raggio di pochi chilometri, il territorio era la rete di prossimità tra i produttori e gli users dei loro prodotti, ambedue legati direttamente o indirettamente alla "terra". Al tempo, il territorio, diventando il contesto di esperienza condiviso da tutti gli operatori locali, lontano o estraneo rispetto a tutti gli "esterni”, plasmava, di fatto, la società locale, sedimentazione delle esperienze comuni vissute nel luogo.

Questa forma di identificazione dei diversi luoghi, tramite l'appartenenza a reti di prossimità e a società locali differenti, ha avuto un ruolo fondativo nell'organizzare i rapporti tra i sistemi locali durante tutta la storia pre-moderna. Nel paradigma tradizionale (pre-moderno) di produzione e di convivenza sociale, i soggetti presenti in ogni luogo, sono immersi in un contesto che essi non hanno scelto, ma è stato prodotto dall'evoluzione passata. E non possono distaccarsi più di tanto dalla sua cultura e dalle sue regole, perché i fattori produttivi - la terra, il lavoro manuale, il capitale disponibile - sono fattori relativamente immobili, che tendono a rimanere dove la natura e le vicende della storia li hanno collocati. Dando

\footnotetext{
Professore di Economia della Conoscenza presso il TeDIS Center - Venice International University

e-mail: enzo.rullani@gmail.com
}

sinergie, rivista di studi e ricerche

n. 91, Maggio-Agosto 2013, p. 141-163

ISSN 0393-5108 - DOI 10.7433/s91.2013.08 
luogo, così, a sistemi tendenzialmente chiusi e abbastanza stabili (salvo guerre e carestie), in cui ciò che accade all'esterno ha poca capacità di influenza.

La tradizione dei borghi e delle città storiche, in Italia, ha conservato - per certi aspetti - questa antica eredità "municipale" e locale. Qualche volta, questa eredità locale è diventata localistica, nel senso che si è fatta ideologia differenziale, portata avanti con orgoglio in contrasto agli "odiati vicini" e alle alternative esterne. Ma, nella maggior parte dei casi, la modernità ha recuperato questa tradizione dando ai sistemi locali riferimenti esterni diversi, e più forti, tali da cambiare anche la natura della coesione interna e il baricentro dell'organizzazione nelle società locali.

\section{La prima modernità livella il territorio, riducendolo ad uno spazio piatto}

Rispetto all'idea tradizionale di territorio, la modernità che irrompe negli assetti pre-moderni a partire dalla rivoluzione industriale di fine settecento, modifica gli assetti precedenti perché si appoggia ad un fattore mobile, trans-territoriale: la conoscenza tecnica (codificata, replicabile) che sta nelle macchine, e che propone un modo di produrre astratto, che riduce il lavoro a tempo-lavoro (privo di connotazioni locali), il capitale a capitale-denaro (anch'esso indifferenziato) e il territorio ad un contenitore vuoto (un certo numero di metri quadrati e una certa rete di strade ed edifici di servizio, rispetto alla fabbrica).

La diffusione pervasiva della produzione a macchina di prodotti industriali introduce due importanti fattori di mobilità trans-territoriale, che caratterizzano il paradigma produttivo (capitalismo mercantile) emerso con la rivoluzione industriale e propagatosi poi in tutta Europa nel corso dell'ottocento:

- la macchina (o meglio la conoscenza codificata contenuta nella macchina) è un

fattore produttivo trasferibile, che - come tale - attraversa i territori e non resta "confinato" al luogo di origine;

- il prodotto industriale, ottenuto con tecniche standard, che non dipendono dalla localizzazione, risponde a una domanda che è, anch'essa, trans-territoriale.

Tecnologia e capitale, e qualche volta anche l'imprenditorialità necessaria per attivarle, vengono spesso da fuori, ossia dalle aree più sviluppate del tempo, nel momento in cui le imprese e le persone che stanno sulla frontiera della rivoluzione industriale vanno ad applicare il loro sapere ad aree in cui sono disponibili fonti di energia (fiumi, carbone) e forza lavoro a basso costo.

In questa nuova condizione, sia la macchina (e spesso anche il capitale e il sapere per utilizzarla) che i componenti (a monte) e i prodotti (a valle) non restano chiusi nel ristretto circuito locale, ma fanno riferimento ad un circuito esterno sempre più rilevante; inoltre, al termine del ciclo produttivo, una parte rilevante dei prodotti ottenuti dalla fabbrica locale cercano sbocchi di mercato in altri luoghi.

Le barriere fisiche e politiche tra i tanti sistemi locali che frazionavano l'economia pre-moderna vengono così abbattute nel corso dell'ottocento, quando si sviluppano i nuovi mezzi di trasporto (treno, ferrovia, navigazione a vapore) e 
prendono forma - un po' dappertutto - Stati nazionali estesi, per effetto delle rivoluzioni borghesi, impegnate ad unificare il mercato ad una scala più grande di quella locale.

Nel capitalismo dell'ottocento, si crea così un nuovo amalgama: l'economia esce dai confini locali (municipali, cittadini) e diventa economia nazionale, aperta alle grandi aree nazionali presenti in Europa (inglese, francese, tedesca, italiana), con le loro proiezioni imperiali in altri continenti. Il territorio inteso come sintesi di società locale e di sistema produttivo locale (di prossimità) perde peso, a favore del territorio inteso come sintesi di società nazionale e istituzioni statali, basate sul mercato nazionale e sulle regole standard create dallo Stato di diritto (leggi astratte e generali), che tolgono rilevanza alle differenze tra i luoghi. È il momento in cui molti lavoratori della terra vengono "proletarizzati" e costretti a confluire nelle città industriali, ingrossando le fila di una classe operaia che offre lavoro generico, indifferenziato, non potendo utilizzare le abilità accumulate in precedenza nel lavoro dei campi. Ed è anche il momento in cui i prodotti e le condizioni di vita si standardizzano, perdendo contatto con le storie precedenti dei luoghi.

Il territorio finisce così per perdere, nel contesto della modernità industriale, quella grana fine che differenziava i luoghi e rendeva i loro abitanti diversi da quelli presenti in altri luoghi, essendo ciascuno portatore di una cultura e di capacità professionali aderenti alle qualità distintive del luogo di origine. L'origine perde valore, di fronte agli standard produttivi dettati da una tecnologia priva di qualificazioni storiche e geografiche. Cosicchè il passato implode in un presente tendenzialmente privo di forti qualificazioni territoriali, essendo le ultime rimaste destinate - si pensava - a perdere progressivamente di rilevanza dal punto di vista della produzione.

\section{La riscoperta del territorio nel fordismo}

Tuttavia, l'oblio del territorio, generato dalla modernità, non dura molto. Va avanti per tutto l'ottocento, ma comincia a perdere vigore con l'avvento del nuovo secolo. Nel novecento, infatti, le qualità differenziali del territorio vengono progressivamente recuperate dallo sviluppo del paradigma fordista, che ha il suo baricentro non più nella macchina (astratta), ma nell'organizzazione, che combina molte macchine in modi di volta in volta differenti, aderenti al contesto e al fine perseguito.

Le organizzazioni che prendono il comando del modo di produzione fordista non sono frutto di un calcolo ingegneristico di ottimizzazione tecnica (come le macchine). Al contrario, ciascuna grande impresa emerge dall'esperienza pratica, seguendo percorsi che non sono disegnati a tavolino, ma che prendono forma sulla base di decisioni ad hoc e di modelli organizzativi e gestionali differenti. In tal modo, ciascuna grande impresa si qualifica come unica, rispetto a tutte le altre, essendo frutto di un apprendimento evolutivo che procede per prova ed errore, e va avanti a zig zag, cercando sperimentalmente il modo di risolvere i problemi che di 
volta in volta sorgono lungo il cammino intrapreso. Del resto, questo è l'unico modo per affrontare la complessità di organizzazioni che devono governare decine di fabbriche, migliaia di dipendenti, schiere di "commerciali" che vanno alla ricerca di sbocchi su mercati sempre più vasti e differenziati.

Cambia anche il modello di razionalità adottato, perché il calcolo dell'ottimo, a tavolino, può andare bene per la risoluzione di un problema tecnico o gestionale a complessità ridotta, ma non funziona se l'organizzazione prende forma attraverso un approccio sperimentale, ben descritto da Simon H. (1982), per cui si fanno dei tentativi e si selezionano quelli che danno risultati soddisfacenti, sfuggendo così al determinismo del calcolo ex ante. Il risultato è che ciascuna impresa segue un percorso che è osservabile solo ex post, e il risultato di questo percorso è che la sua forma organizzativa, la sua cultura aziendale, il suoi prodotti e persino i suoi lavoratori finiscono per diventare firm specific, diversi da quelli presenti in ogni altra impresa, anche dello stesso settore e dello stesso luogo. Non è un caso che le aziende emerse nel paradigma fordista, un po' come le persone, cominciano ad essere identificate con un nome unico, che dice molto di più di qualificazioni astratte come la dimensione, il settore di appartenenza, la localizzazione, i prodotti offerti al mercato. La Fiat che prende forma a Torino, per fare un esempio, non è solo un'azienda grande, del settore automobilistico, che opera nel milieu torinese e che fa automobili in prevalenza utilitarie. È molto di più, avendo qualità personali, esperienze e relazioni col contesto che sono uniche, e che per capire il suo comportamento e i suoi problemi bisogna conoscere, perché ne fanno un attore distinto da tutti gli altri nel gioco competitivo.

Il territorio in cui si localizza una grande impresa fordista (Torino, nell'esempio fatto) diventa anch'esso firm specific, e dunque diverso da ogni altro, perché la grande organizzazione con il suo indotto definisce l'asse che dà forma alla rete produttiva locale e struttura la società locale. Tornano nuovamente ad essere importanti le città (un po' più grandi dei borghi pre-moderni) e il loro immediato hinterland.

Gli Stati nazionali mantengono il loro ruolo unificante sul piano istituzionale, ma non governano più su mercati e interessi privi di identità locali. Al contrario, gli Stati nazionali federano, per così dire, una pluralità di imprese che rappresentano e "difendono" gli interessi del loro indotto, ossia della società e della rete produttiva locale con cui vivono in simbiosi.

Questa storia di rafforzamento delle identità territoriali in chiave moderna continua e si rafforza quando il fordismo comincia a manifestare i primi segni di crisi (anni settanta) lasciando il posto ad un nuovo paradigma: il capitalismo della flessibilità (1970-2000), che in Italia si organizza in base a sistemi produttivi popolati dall'impresa diffusa: migliaia di piccole e piccolissime imprese emerse dal basso, che - nel caso dei distretti industriali - si addensano in clusters locali specializzati, ciascuno, in uno specifico settore produttivo. Per cui a Prato si fanno i tessuti e tutti si occupano di tessuti, mentre a Manzano si fanno le sedie e tutti si occupano di sedie. Il collante di prossimità diventa il collante produttivo più 
importante dal punto di vista della produzione di valore e della competitività, e questo consolida il ruolo svolto dalla cultura e dalle istituzioni della società locale.

Accade così che, nei trenta anni che precedono il cambiamento di secolo, il territorio - inteso come sintesi di rete produttiva e società locale - torna ad occupare il centro della scena, almeno in Italia. In esso si fanno le economie di scala e di specializzazione (visto che le singole imprese sono troppo piccole per operare da sole), in esso nascono le nuove imprese, in esso si fanno e si imitano migliaia di micro-innovazioni ogni anno, in esso si forma una professionalità del lavoro e una cittadinanza istituzionale che vivono in simbiosi colla specializzazione settoriale prescelta. Il territorio, in altre parole, diventa il vero organismo produttivo, prendendo il posto che era stato della grande organizzazione fordista.

Ma nel decennio che inaugura il nuovo secolo, negli anni post-2000, siamo di nuovo ad una svolta. Per una serie di circostanze (globalizzazione, ICT), comincia a prendere forma un modo di produzione (il capitalismo globale della conoscenza) che usa le filiere globali per sfruttare le grandi differenze di capacità e di costi presenti in un mercato mondiale che si sta sempre più unificando. Di conseguenza le conoscenze che si accumulano in modo progressivo nei singoli luoghi di esperienza non bastano più: una parte di esse deve essere trasformata per divenire mobile, in modo da dare vita a iniziative produttive moderne anche in luoghi diversi e lontani da quelli di origine. La risorsa critica per competere, in questo nuovo paradigma, non è più la localizzazione nel singolo territorio, ma la creazione di una rete multiterritoriale, che specializza le funzioni svolte da ciascun segmento locale nella filiera globale.

Le imprese che prima facevano parte del sistema produttivo locale, cominciano ad alzare la testa e guardarsi intorno, scoprendo convenienze e possibilità fuori del territorio di origine. Col risultato di affiancare alle radici territoriali (che rimangono) una rete sempre più fitta di collegamenti, specializzazioni, scambi che invece le mettono in relazione con interlocutori (fornitori, centri di ricerca, committenti, distributori, consumatori) che stanno altrove.

Il valore è co-prodotto da un nuovo organismo produttivo, la filiera multiterritoriale, che si avvia a diventare tendenzialmente globale.

\section{Una diversa idea di territorio}

Per quanto detto, a partire dal 2000, il concetto di territorio cambia in profondità.

Il territorio, nel novecento, era infatti, come abbiamo detto, un sistema compatto di esperienze e di culture cumulate localmente, con la mediazione del comando della grande impresa o con quella della prossimità distrettuale.

Ora non è più un sistema compatto, e forse non è più nemmeno un sistema nell'accezione tradizionale del termine.

Ogni luogo, ospita, infatti al suo interno spezzoni di reti produttive e di società locale (frammentata), ma ospita anche nuclei di attività e interessi che si rifanno a filiere esterne, e che poco hanno a che vedere con coloro - imprese o persone - che 
lavorano o vivono nella stessa strada, nello stesso quartiere, nello stesso centro urbano.

In questo modo, il territorio si frammenta, perché le persone e le imprese che si trovano in ciascun luogo non fanno più riferimento ad un comune circuito di prossimità, ma cominciano ad avere ciascuna prospettive, rapporti, interessi differenti.

Per due ragioni di fondo.

Innanzitutto, il singolo territorio perde la sua autonomia perché viene a dipendere - per la sua funzione di generazione di valore - da ciò che accade in altri territori, che sono attraversati dalla stessa filiera. Si crea una situazione di interdipendenza con attori e comportamenti situati all'esterno, e spesso molto distanti. Ogni luogo diventa in questo modo una tessera di un mosaico più grande, che ne detta la funzione e il significato.

Inoltre, quello che resta del sistema locale può facilmente diventare - anche in ragione delle reti globali che lo attraversano - un insieme differenziato e caotico di parti in conflitto tra loro, debolmente unite dal rapporto di prossimità e da una comune identità territoriale. Non è detto che accada, ma questa deriva verso l'ingovernabilità è esperienza comune di molti luoghi, anche se può essere contrastata e "ricucita" dai soggetti direttamente interessati.

\section{Lo tsunami in corso: la conoscenza replicativa diventa mobile e alimenta la trans-territorialità delle reti globali di produzione}

Nelle filiere globali, la conoscenza impiegata nella produzione subisce una torsione che ne separa due nuclei fondamentali:

- la conoscenza generativa (conoscenza che produce altre conoscenze, innovando, adattando, sperimentando, immaginando ecc.) rimane localizzata perché essa è collegata alle persone e ai contesti in cui ha preso forma, e può essere solo lentamente e con molte difficoltà riprodotta e trasferita altrove (per essere impiegata da altre persone e in altri contesti);

- la conoscenza replicativa, invece, trova nelle filiere globali la condizione per il suo massimo sviluppo. Essa è infatti quel tipo di conoscenza - astratta, impersonale - che viene prodotta in forma codificata apposta per renderla trasferibile e riproducibile in luoghi diversi da quello di origine, in modo da poter sfruttare sia le grandi differenze di costo e di capacità cui le filiere globali danno accesso, nel mondo, sia $\mathrm{i}$ grandi moltiplicatori che discendono dall'allargamento del bacino potenziale di uso a miliardi di consumatori.

La globalizzazione dei mercati rende estremamente conveniente ricavare dalla conoscenza generativa posseduta dei modelli o delle soluzioni codificate (tecnologie, macchine, prodotti standard, software, algoritmi) che sono applicabili ovunque, e da qualsiasi persona, purchè rispetti le "istruzioni per l'uso" fissate dal codice. Si pensi solo a quello che è accaduto - e accade oggi - nei rapporti tra la 
Cina, da una parte, e quelle che, alla fine novecento, erano le due maggiori potenze industriali del mondo, Stati Uniti e Giappone.

Dal 2000 al 2011, in soli undici anni, la quota della produzione manifatturiera mondiale detenuta dalla Cina è passata dall' $8,3 \%$ al $21,7 \%$ (seguita, a ruota, dagli altri paesi dei BRICS: Brasile, Russia, India, Sudafrica, oltre alla Cina stessa) ${ }^{1}$.

Bisogna tenere presente, per capire la rapidità dello tsunami in corso, che la quota della Cina arrivava soltanto al 4,1\% all'inizio degli anni novanta: meno di quella dell'Italia. Non era un secolo fa, ma solo venti anni fa.

Il che vuol dire che in ognuno di questi venti anni più dell' $1 \%$ delle attività mondiali si è spostato regolarmente verso la Cina, per effetto dei differenziali di crescita. Una cifra imponente. Certo, la rapidità del cambiamento è stata favorita dalle scelte di delocalizzazione e di investimento diretto delle multinazionali americane e giapponesi (o degli altri paesi occidentali), che hanno cercato, in questo modo, di catturare il "surplus" associato alla differenza abissale nei costi del lavoro tra le precedenti e le nuove localizzazioni. Ma, anche a prescindere dalle multinazionali, il massiccio trasferimento in corso è dovuto a due dati strutturali di fondo: la nuova mobilità delle conoscenze (che si codificano, diventando astratte $\mathrm{e}$ mobili rispetto al luogo di origine) e la crescita dei flussi commerciali che vengono liberalizzati a scala mondiale.

Mentre, dal 2000 al 2011, la Cina triplicava il suo peso nella produzione manifatturiera mondiale, la quota degli Stati Uniti passava dal 24,8\% al 14,5\% (era del 21,8\% nei primi anni novanta, prima del boom della new economy internettiana).

Il crollo della quota prodotta dal Giappone, nello stesso periodo, è anche più profondo e duraturo: dopo essere arrivata al 19,4\% nei primi anni novanta, per effetto del decennio d'oro dell'espansione giapponese negli anni ottanta, essa scende prima al 15,8\% nel 2000 e poi al 9,4\% nel 2011. Meno della metà, in venti anni.

I numeri parlano da sé: la parte della manifattura che negli Stati Uniti e nel Giappone si trova ad essere non più competitiva - nelle filiere globali che prendono corpo col nuovo secolo - viene delocalizzata o semplicemente chiusa, e sostituita da importazioni di beni intermedi o finali dai paesi low cost. Nelle produzioni a monte delle filiere, siamo arrivati a valori non immaginabili fino a pochi anni fa: nel 2012 la produzione cinese di acciaio (716 milioni di tonnellate) arriva a volumi che sono quasi dieci volte (dieci volte!) quelli degli Stati Uniti (89) e sette volte quelli del Giappone $(107)^{2}$. Questo divario ha preso corpo in una situazione in cui la Cina non è ancora, nel suo insieme, un paese realmente industrializzato, se si tiene presente

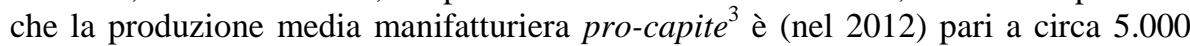
dollari: sei volte meno della Germania (30.000 dollari), cinque volte meno del Giappone (25.000), tre volte meno dell'Italia e degli Stati Uniti.

Che cosa dobbiamo aspettarci per il futuro, quando il prodotto manifatturiero pro-capite della Cina salirà, seguendo il percorso già visto, magari con tassi meno

\footnotetext{
Elaborazioni CSC su dati Global Insight.

Fonte: World Steel Association.

Fonte: elaborazioni CSC su dati Global Insight.
} 
eclatanti ma comunque significativi? E che cosa succederà quando l'India - altro gigante che si sta muovendo a passi accelerati verso l'industrializzazione accrescerà il suo prodotto manifatturiero pro-capite che oggi è meno di 1.000 dollari, ossia sei volte meno di quello della Cina, trenta volte meno di quello della Germania?

È un cambiamento di fondo, che segnerà sicuramente i prossimi decenni del secolo, in ciascun paese, compresa l'Italia. Portando ad una concezione del territorio che dovrà cominciare a vederlo come parte di filiere globali che ad esso delegano funzioni e competenze specializzate, più o meno importanti, in una logica di divisione del lavoro che valorizza alcune qualità dei contesti locali, mentre svalorizza o rende irrilevanti altre.

Il paradigma territoriale, insomma sta nuovamente cambiando per alcuni dati di fondo. Rifarsi a concezioni territoriali che risalgono al fordismo o al capitalismo distrettuale del secolo scorso rischia di mettere in ombra le trasformazioni che stanno avvenendo nel presente e soprattutto le opportunità e minacce di evoluzione che avremo di fronte nel prossimo futuro.

Il punto essenziale è che oggi conoscenze generative e conoscenze replicabili tendono a separarsi e distanziarsi, connettendosi a distanza entro le reti e le filiere globali. La loro sintesi non è più nel singolo territorio, ma nel rapporto transterritoriale che attraversa ciascun luogo, collegando i sistemi produttivi locali con flussi di conoscenze, merci, capitali sempre più rilevanti.

Infatti, nel fordismo e nel capitalismo distrettuale del novecento, le imprese e le persone impegnate nella produzione fornivano e usavano, insieme, conoscenze generative e replicative nello stesso luogo, essendo esse quasi sempre integrate l'una con l'altra nella testa delle persone, nel sapere dell'organizzazione o nel sistema di flussi a breve distanza che circolavano nelle reti locali.

L'impresa fordista che standardizzava e cercava di raggiungere i più grandi volumi possibile, usando soprattutto conoscenza replicativa, è la stessa impresa che all'occorrenza - era capace di modificare macchine, programmi, rappresentazioni, calcoli per adattarli a contesti geografici e personali nuovi, mettendo in campo il sapere generativo di managers, ricercatori, uomini del marketing, comunicatori.

Lo stesso accadeva nella tipica impresa artigiana del capitalismo distrettuale: da un lato, l'artigiano cercava di replicare al massimo le soluzioni che funzionano e fanno vendere, ma - nel caso servisse - era anche in grado di usare le conoscenze generative possedute per cambiare le soluzioni da applicare.

Nella filiera globale post-2000, invece, questa simbiosi (personale/organizzativa) tra conoscenza generativa e conoscenza replicativa si rompe, diventando un'eccezione e non più una regola: in certi territori si fanno le cose nuove e difficili, impiegando nuclei di conoscenza generativa (ad alto costo e ad alto valore aggiunto), mentre in tanti altri, a cui sono stati delegati compiti esecutivi ben codificati o meccanizzati, ci si limita ad usare conoscenza replicativa, associata al lavoro e a contesti low cost.

In ciascun territorio viene in questo modo alterato l'equilibrio tra conoscenze generative e replicative, specializzando le parti. È naturale che nei territori high cost 
(ricchi) possano rimanere solo conoscenze generative, che è difficile trasferire o riprodurre altrove. Le conoscenze replicative - le macchine, i prodotti, le soluzioni codificate che sono prodotte dalle conoscenze generative - subiscono invece 1" "attrazione fatale" dei paesi emergenti, dove si possono trovare fattori low cost, guadagnando la differenza di costo relativa.

\section{II territorio nelle filiere globali: una sfida dall'esito ancora incerto}

Ogni territorio viene in questo modo ridefinito dal fatto di essere inglobato in una logica di filiera globale. I cambiamenti di fondo, di cui dobbiamo prendere atto, sono soprattutto due:

a) L'economia globale, unita con l'uso pervasivo delle ICT, ha ridotto le distanze tra i luoghi, inserendoli in filiere mondiali che ne valorizzano la specializzazione e le differenze distintive. Ogni territorio è in questo modo diventato potenzialmente concorrente con tutti gli altri, se offre una competenza o una funzione imitabile o sostituibile; ma anche potenzialmente complementare se si specializza in funzioni e competenze distintive che possono legarlo ad altri territori, nella co-produzione di valore realizzata dalla stessa filiera. Di conseguenza l'identità di ciascun luogo, oggi, comincia ad essere definita in rapporto ai flussi e alle reti che lo legano ad altri luoghi, vicini e lontani, in una condizione di interdipendenza che col suo consolidarsi, finisce per modificare i tratti distintivi di ogni territorio. In passato gli abitanti di un luogo sentivano di appartenere - volenti o nolenti - ad una storia e ad un assetto territoriale, ereditati dal passato, cumulando esperienze, emozioni, relazioni in prevalenza locali. Oggi, accanto a questi elementi distintivi, ciascuna impresa, persona, comunità ha imparato ad ibridare la sua esperienza dei luoghi con l'esperienza fatta nelle reti globali, nei flussi di scambio con l'esterno e in rapporto a quanto di competitivo, coinvolgente, innovativo, accade nel "villaggio globale" in cui si svolge la vita e il lavoro di tutti noi.

b) La competizione/complementarità tra i luoghi attraversati dalle filiere globali decostruisce gli assetti e le culture presenti in ciascun territorio, differenziando interessi, concezioni, destini presenti al suo interno. Ogni territorio comincia infatti ad ospitare non solo parti dotate di strategie e visioni del mondo differenti (cosa che accadeva anche in precedenza) ma parti che si legano a flussi transterritoriali e ad altri luoghi, presenti nelle filiere globali di appartenenza, e che agiscono seguendo logiche e strade che tagliano fuori gli altri, i "vicini" che abitano e lavorano nel circuito di prossimità. Ne deriva un effetto "scompositivo", per cui la prossimità geografica non è più sinonimo di prossimità di cultura, di interessi e di comportamento. Le aziende "deboli" che, sotto la pressione della specializzazione di filiera e della concorrenza, vivono ripiegate su se stesse, rimanendo sulle difensiva, si contrappongono alle aziende "forti", che usano la filiera globale per crescere, liberarsi dai legami pregressi e andare oltre l'orizzonte locale. Quelle che scelgono strategie "responsabili" che 
le legano ai fornitori e dipendenti locali hanno poco da dire alle imprese più opportuniste o più mobili che sfruttano le differenze di costo e di capacità accessibili in altri luoghi. Il territorio, attraverso queste cesure e tensioni interne, diventa un "cantiere" in cui il vecchio edificio viene de-costruito e si tenta - con esiti non sempre felici - di ri-costruirne uno nuovo, coerente con le esigenze e opportunità del contesto globale.

\section{Ma, attenzione: il territorio conta ancora}

Eppure, la prossimità geografica continua a contare, perché non è la stessa cosa operare in Brianza, in Polonia o in California. Istintivamente ciascun di noi dà un significato pregnante a queste qualificazioni, perché sa che esiste una geografia dell'innovazione che muove non solo persone e imprese, ma anche contesti territoriali dotati di precise caratteristiche distintive (Plechero, 2012), che li differenziano da altri.

Il cemento territoriale allenta la sua presa, ma ogni territorio continua ad avere una sua differenza, rispetto agli altri: una differenza che conta. Essa deriva, innanzitutto, dai tratti identitari che rimangono attivi, anche se meno compatti di prima, dopo la decostruzione della cultura e dell'identità ereditate dal passato; ma deriva anche dall'esigenza che i soggetti hanno di stare nel mondo ri-costruendo una (nuova) identità condivisa e riconoscibile, con cui dialogare con gli interlocutori presenti nelle filiere globali di appartenenza.

Chi abita o lavora in un luogo ha infatti la necessità di gestire e arricchire beni comuni di grande rilevanza nella divisione internazionale del lavoro o comunque beni distintivi, intesi come capacità e risorse che, essendo accessibili esclusivamente o prevalentemente a chi abita o lavora in un luogo, lo qualificano come differente da altri:

- il circuito di conoscenza generativa, che nasce dall'interazione di prossimità e da quella sviluppata nello spazio metropolitano;

- la rete di relazioni che allaccia e specializza le diverse unità del cluster territoriale;

- i centri di ricerca, educazione, sperimentazione, servizi che facilitano il lavoro e l'interazione a scala locale;

- l'accesso alle grandi piattaforme logistiche e comunicative (ICT) che collegano il luogo col mondo;

- il nucleo di significati ed esperienze distintive che, sintetizzato in un brand, rende riconoscibile un luogo nei sistemi commerciali e comunicativi mondiali

Tutte queste risorse chiave - che non hanno natura proprietaria, ma sono beni comuni o parzialmente condivisi - hanno una grande importanza nella divisione internazionale del lavoro di oggi, e spiegano perché le imprese, pur operando in filiere trans-territoriali continuano ad avere radici in questo o quel luogo. Ma si tratta di beni che non possono essere semplicemente ereditati dal passato, perché sono frutto degli investimenti pluriennali fatti allo scopo. Se l'identità di un territorio e i 
motivi della condivisione del luogo si attenuano, scendendo oltre una certa soglia, diventa difficile o anti-economico, per i privati, fare investimenti che andrebbero, in una misura più o meno rilevante, a vantaggio di altri, che non partecipano ai costi e ai rischi dell'operazione, ma - grazie alle relazioni di prossimità - ne possono intercettare i benefici, quando questi cominceranno a venire.

Dunque, la ricostruzione di una identità territoriale non effimera, capace di alimentare l'investimento condiviso nei beni comuni (o distintivi) è un traguardo importante, anche se difficile, nell'evoluzione dei diversi territori.

Il territorio diventa, come si è detto, un sistema incompiuto (Rullani, 2010), ossia un sistema che cerca continuamente di rigenerare la propria identità in rapporto agli eventi esterni che la de-costruiscono, ma che - per varie ragioni - non ha il potere e in parte la volontà di chiudere il cerchio. I suoi feedback non riescono mai a ricostruire compiutamente il sistema e la sua identità, ma lasciano aperti varchi per sperimentare soluzioni nuove e impreviste, facendo posto alla creatività dei soggetti e alla serendipity del caso.

Un sistema incompiuto non è un sistema chiuso in sé stesso e replicativo; ma, al contrario, è un sistema in transizione che, grazie all'intelligenza generativa dei soggetti con cui ha rapporto riesce a trasformarsi per assumere forme nuove, capaci di rispondere alle opportunità e alle sfide di ogni stagione.

\section{Territori in transizione}

Per quanto detto, il territorio, oggi, è una realtà (e un concetto) in transizione, perché deve ridefinire la propria funzione e identità in rapporto ad un mondo esterno che sta cambiando in modo rapido e radicale. Stiamo assistendo ad una trasformazione dei ruoli e delle competenze nell'economia mondiale di proporzioni mai conosciute in precedenza: fenomeno assolutamente nuovo di cui non sappiamo gli esiti futuri e che sta sconvolgendo l'organizzazione interna di tutti i territori.

Un flusso imponente di conoscenze codificate sotto forma di macchine, di software, di scienza, di tecnologia, sta passando dai Paesi ad alto costo come il nostro, ai Paesi a basso costo che stanno nel mondo.

Questa trasformazione fa si che una parte delle nostre produzioni radicate territorialmente che hanno alle spalle una tradizione verrà svalorizzata e piano piano, o si sposteranno, o non si guadagnerà con queste cose.

Questo poi pone a noi la sfida, noi che in qualche modo in questi territori rimaniamo, di trasformare la nostra base cognitiva: noi abbiamo bisogno, man mano che le conoscenze codificate e quindi trasferibili, impersonali, vanno in questi Paesi dove i fattori costano meno, che il nostro lavoro, le nostre persone, le nostre imprese, i nostri territori diventino dei contenitori di conoscenza generativa, non di conoscenza codificata, perché quello che non è mobile è ciò che resta attaccato alla testa della gente e quindi alla testa delle organizzazioni che mettono insieme le persone, all'intelligenza fluida, come dire, che è informale e quindi difficilmente trasferibile. 
Il compito che le imprese e i territori devono affrontare nel nostro secolo, per compensare i differenziali di costo con cui ci presentiamo alla concorrenza internazionale è difficile e di grande portata. Bisogna infatti:

- trasformare gran parte delle nostre organizzazioni, delle nostre imprese in produttori di conoscenza generativa e accumulare in loco questo tipo di conoscenza con un flusso consistente di investimenti nel nuovo;

- usare la conoscenza generativa disponibile, nella forma specifica presente in ogni luogo, per mettere a punto modelli originali e innovativi di macchine, prodotti o altri artefatti replicativi che possano avere un bacino di vendita o di uso molto ampio, associato a grandi moltiplicatori di valore.

I nostri territori oggi sono in ebollizione per questo: una parte del territorio (la sua tradizione, la sua storia) sta morendo, mentre un l'altra parte sta nascendo o cambiando in profondità rispetto agli assetti pre-esistenti. Ma, perché questo processo di selezione e rafforzamento competitivo vada avanti, senza perdite di posti di lavoro e di reddito, bisogna superare molti ostacoli.

\section{II dibattito al convegno di Sinergie}

Se questa è la premessa, credo che si possano trarre alcune conclusioni dal dibattito che è nato sulle forme assunte dai territori in transizione, sollevando tre problemi chiave:

- un problema di metodo, in merito ai modelli teorici da impiegare per descrivere il processo di innovazione che avviene nel circuito micro-macro (ossia impresaterritorio);

- un problema di contenuti, che riguarda invece la natura delle forze che portano avanti il processo di innovazione ai due livelli (micro e macro);

- un problema di qualità, che cerca di definire la funzione oggi assolta dalla prossimità territoriale in un sistema produttivo che è diventato più mobile $\mathrm{e}$ multi-territoriale.

Vediamo questi tre punti, usando il contesto interpretativo proposto sin qui per rileggere quanto è stato detto al convegno.

\section{Un problema di metodo: il puzzle micro-macro per chi studia l'impresa}

Il primo punto che è emerso nella discussione, è un problema di metodo. Che metodo usiamo per capire le forme della transizione territoriale?

Ci si è chiesti, ad esempio, quali siano i modelli più adatti per analizzare la relazione circolare micro-macro. In certi casi, infatti è l'impresa che genera, con le sue scelte, la trasformazione del territorio (o dei territori) in cui opera. In altri casi, invece, sono i cambiamenti che intervengono nel territorio ad innescare le trasformazioni osservabili nelle imprese. In generale, la relazione vale nei due sensi, 
ma i modelli teorici che descrivono i processi studiati dalla nostra disciplina possono - implicitamente, e dunque non consapevolmente - privilegiare l'una o l'altra catena causale.

Essendo noi studiosi dell'impresa, tendenzialmente saremmo portati a dire che ciò che cambia a livello macro, diventando visibile ad un certo momento, è (sicuramente) stato innescato dalle innovazioni pionieristiche di alcune imprese che, col loro successo, avviano la trasformazione di tutto il sistema, perché - in presenza di una soluzione che rappresenta la one best way - le altre imprese o si adeguano (imitando) o chiudono.

Ma questo "pregiudizio disciplinare", per così dire, non ci fornisce una chiave efficace per trattare il problema della relazione micro-macro.

Le teorie evoluzionistiche, che hanno trovato spazio anche entro la cornice della teoria dell'impresa, sembrano a prima vista convalidare questo approccio, fissandolo in uno schema darwinistico di prevalenza del "più adatto" che, in termini competitivi, diventa il "migliore", il "più innovativo". Ma si tratta di una validazione più apparente che reale.

La teoria evolutiva, infatti, da sempre, e in tutte le discipline a cui è stata applicata, fa nascere il nuovo da varianti che sono più o meno casuali, che vengono selezionate dall'ambiente e, se vincono nel rapporto competitivo con le altre, si propagano, modificando il contesto macro. Quindi abbiamo una corrispondenza causale tra il singolo evento (nel nostro caso, l'impresa che sfrutta un'idea nuova), e il contesto che viene modificato, se questa idea si rivela "vincente". Naturalmente se, al contrario l'idea nuova non emerge o - per varie ragioni - non viene portata avanti, il contesto, in mancanza di "capitani coraggiosi" diventa conservatore. Per cui, seguendo questa intuizione, c'è bisogno sempre di un pioniere (impresa) e di una nuova idea (innovazione) perché il contesto macro possa cambiare.

È un modello che la teoria evolutiva propone da sempre. Ma se approfondiamo la questione, ci accorgiamo che questo modo di ragionare ci fornisce solo delle soluzioni ex post. Guardando i cambiamenti che emergono nei territori, dovuti ad eventi che sono maturati nel contesto micro ma di cui abbiamo perso la traccia, possiamo solo prendere atto del fatto che si sono evoluti e sono diventati così, probabilmente perché qualche impresa ha avviato il processo e le altre l'hanno seguita. Ma lo constatiamo ex post, a cose fatte.

Questa convinzione ci può servire fino ad un certo punto quando la trasportiamo sul terreno metodologico dell'economia di impresa e del management del suo cambiamento. Infatti, il processo decisionale dell'impresa, che è oggetto dei nostri studi, è una costruzione ex-ante: l'impresa cerca di prefigurare quello che essa può fare di nuovo e cerca di trasformare il suo contesto territoriale in modo coerente con le sue strategie. In questo caso, la relazione micro-macro rimane in piedi, ma va vista in chiave di costruzione del futuro possibile e desiderato, non di evoluzione che si rivela ex post come l'unica possibile, ma a cose fatte.

Se la teoria evolutiva ci offre una soluzione che è solo in parte soddisfacente, dobbiamo pensare ad un'altra idea. Nel momento in cui i protagonisti micro sono uomini e non amebe o uccelli darwiniani, entra in campo una forza nuova: 
l'intenzionalità. Ossia la capacità di innovare, di inventare, di immaginare, di convincere. E questo cambia tutto.

L'impresa che, intenzionalmente, innova, propone una soluzione fuori standard, lancia un'idea originale, altera il processo evolutivo che - se lasciato a sé stesso e alla causalità delle varianti - sarebbe lento, dispersivo. Quell'impresa ha messo in moto un'altra dinamica, avviando un processo auto-organizzatore in cui le persone si danno da fare, sono proiettate sul futuro, creano dei significati, danno senso alla propria visione del mondo, e la traducono in sogni o racconti capaci di emozionare, convincere. Tutto il convegno di Sinergie è stato costellato da esortazioni a cambiare il significato delle cose, a raccontare in un altro modo quanto si fa, a cambiare l'identità delle persone che vivono il territorio. Questo è un processo che non è semplicemente leggibile in chiave di evoluzionismo. Bisogna invece considerarlo con un'altra chiave metodologica: il costruzionismo. Questo, nelle sue varianti, fa perno su un processo di costruzione del futuro, da parte di soggetti che partono da un proprio progetto di vita o di lavoro. Un progetto che va oltre l'attesa - passiva o rassegnata - che il mondo cambi ed evolva per conto suo, lasciando a noi solo il compito di prevedere i suoi trend "oggettivi" o di constatarli ex post.

Invece, l'effetto macro viene visto da ciascun soggetto - individuale o collettivo come effetto di una propria attività di costruzione più o meno consapevole ma comunque intenzionale, magari accompagnata dalla serendipity, che dà valore a eventi non voluti o dalle conseguenze non previste, non controllate. Tuttavia alla base c'è un processo intenzionale, in cui i soggetti giocano il ruolo di attori intenzionali.

Come è stato detto al convegno, tuttavia, non basta guardare a ciò che gli attori "vogliono fare" o "non vogliono fare". Una logica soggettivistica che rimanda tutto alle intenzioni dei soggetti e alla loro capacità di realizzarli, è anch'essa insufficiente, per chi studia la relazione micro-macro dal punto di vista delle imprese. Le iniziative puramente soggettive possono andare in tante direzioni diverse, ma alcune di esse risultano, alla prova dei fatti, puramente velleitarie; altre sono estemporanee e puntano sulla buona stella del proponente o sulla buona congiuntura del momento. Altre ancora risultano vincenti, ma grazie agli effetti (fuori programma) della serendipity.

\section{Surfing: catturare l'energia delle onde che alimentano la transizione da un paradigma ad un altro}

Dunque, partire dalle capacità e intenzioni soggettive, va bene. Ma la domanda che dobbiamo porci è: qual è l'energia che permette ad alcune, tra le iniziative prese, di attecchire, crescere e integrarsi tra loro o con l'evoluzione del contesto?

C'è un convitato di pietra che abbiamo appena nominato in questo convegno e che, invece, da un po' di tempo ha un grande peso negli eventi e nelle strategie che prendono forma nelle imprese: il cambiamento del paradigma dominante, intervenuto con l'avvio del nuovo secolo, a partire, diciamo, dal 2000. 
È la forza del cambiamento di paradigma - ossia della transizione di cui abbiamo parlato - che permette ai soggetti innovatori di farsi sistema, integrando le loro micro-innovazioni, e, per questa via, modificare il mondo partendo da idee limitate e parziali, che da sole non avrebbero mai la massa critica per cambiare gli assetti macro.

Questa energia non ce l'hanno i soggetti (da soli), ma - per entrare in gioco nella transizione deve appoggiarsi alla forza delle onde che hanno sommerso il paradigma in declino e fatto emergere il nuovo paradigma.

Ogni paradigma parte da alcune innovazioni o novità di fondo, che ci mettono anni per produrre i loro effetti, rendendo convenienti le progressive trasformazioni/innovazioni realizzate dai singoli soggetti. Il fordismo non ci sarebbe stato se prima non ci fossero state tante circostanze che permettevano a Henri Ford di inventare un nuovo modo di organizzare la fabbrica e le lavorazioni in linea in essa realizzate.

Per chiarire meglio questo esempio, bisogna avere in mente che la produzione in linea, che emerge come idea portante della rivoluzione produttiva avviata nei primi decenni del novecento, è una innovazione d'uso che può materializzarsi in quegli anni perché usa le possibilità messe a disposizione da una rivoluzione tecnologica a lungo incubata e alla fine realizzata nei decenni precedenti: la rivoluzione energetica che porta all'uso diffuso e pervasivo dell'elettricità e del motore elettrico. È questa l'onda che fa emergere il nuovo paradigma, destinato a dominare il secolo scorso; ed è alla sua energia che si appoggia l'idea innovativa di Ford.

L'invenzione dell'energia elettrica, e la realizzazione di potenti sistemi di generazione e di trasmissione, che hanno reso l'energia trasportabile e frazionabile senza difficoltà, è stata alla base della fabbrica fordista, perché ha permesso a Henry Ford di parcellizzare il lavoro e meccanizzare le singole operazioni elementari, mettendo in sequenza tante lavorazioni e tante macchine quante sono le "parti" che scompongono un compito complesso come quello di produrre e assemblare un'automobile (Di Bernardo e Rullani, 1985; Di Bernardo e Rullani, 1990). Se le parti in cui occorre scomporre il compito - rendendolo semplice, in modo da poterlo affidare a una macchina - sono, per ipotesi 10.000, bisogna mettere in linea 10.000 lavorazioni e 10.000 macchine, tutte sotto lo stesso capannone e collegate alla stessa linea di lavorazione.

Senza energia elettrica questa concentrazione e distribuzione delle 10.000 macchine in sequenza non sarebbe stata possibile senza l'energia elettrica. Se Henri Ford fosse nato cinquant'anni prima, dunque, il fordismo non ci sarebbe mai stato, o forse ci sarebbe stato anni dopo, non appena l'energia elettrica avesse permesso a qualcun altro di scoprire la stessa cosa. E non si sarebbe chiamato fordismo, dunque.

Ecco la chiave delle grandi trasformazioni che spingono in avanti le imprese, aprendo loro spazi macro (potenziali) di grande portata, ma che sono le imprese stesse a dover esplorare con le proprie forze e con le loro iniziative, prese a livello micro. Anche nella transizione in corso si tratta dunque di catturare l'energia delle onde che stanno dando forma al nuovo paradigma emergente. Immaginiamo che le 
imprese di oggi agiscano come il surfista che sale sull'onda (generata dal nuovo paradigma che emerge), e usa la sua energia per salire in alto.

Il surfista non genera il movimento partendo dalla propria (limitata) energia, ma lo appoggia alla forza delle onde che cerca di intercettare e su cui "sale", facendosi trasportare verso l'alto. Tuttavia non si adagia semplicemente su di loro, ma deve essere abbastanza bravo da riuscire ad andare nella direzione scelta da lui, mentre magari la maggior parte degli altri si adegua alla traiettoria disegnata dall'onda stessa.

Fuor di metafora, l'impresa di oggi deve appoggiare le sue strategie sulla forza delle grandi trasformazioni che hanno preso forma nell'ambiente - spesso per cause non imputabili alle imprese stesse - riconoscendole, utilizzandole, facendo in modo che la sua idea si agganci a qualcosa che nasce nel sistema. Contemporaneamente, però, l'impresa deve mantenere la propria differenza distintiva, rispetto agli altri, nella rotta da seguire, in modo da potersi differenziare competitivamente.

È sempre successo nel corso della storia moderna. Col senno di poi, potremmo riconoscere che questo sta accadendo anche nel contesto di oggi. Del resto, ogni volta che è emerso un nuovo paradigma, il rapporto tra impresa e territorio è cambiato, ridefinendo il circuito micro-macro in funzione delle opportunità connesse con paradigma emergente.

Per esempio l'idea del territorio che emerge dallo sviluppo del paradigma del fordismo, è completamente diversa da quella che, in questo convegno, i diversi intervenuti hanno visto all'opera nel presente.

Infatti, per il fordismo il territorio che cos'è? È uno spazio vuoto - privo di tradizioni e di differenze originarie - che la grande impresa ordinerà secondo le sue esigenze e il suo potere di indirizzo e di comando, d'accordo (ovviamente) con gli Enti locali, le forze della rappresentanza e lo Stato. Nella logica impersonale che questo apparato esprime, le singole persone o le loro comunità presenti in ogni specifico luogo sono del tutto irrilevanti. L'impresa che "modernizza" il mondo rendendolo artificiale, coerente con i suoi programmi e con i canoni livellatori della produzione di massa fa a meno delle idee e delle culture ereditate dalla storia sul piano locale. Se, nel periodo 1900-1970, il mondo viene plasmato da innovazioni che si appoggiano all'energia messa a disposizione dal paradigma fordista, il risultato sarà anch'esso impersonale e privo di riferimenti alla precedente storia del territorio. Sostanzialmente il territorio scompare e viene ri-generato in funzione dei disegni della grande organizzazione a cui si affida, diventando un supporto delle strutture artificiali programmate dalle piramidi private e pubbliche del potere organizzato.

Ma questa artificializzazione del territorio - che fa a meno delle persone e della comunità, livellando storie diverse sullo stesso standard - ad un certo punto ha perso la sua energia costruttiva nel momento in cui gli automatismi creati dal paradigma fordista si sono impigliati nella complessità da esso stesso creata.

Per gestire questa la complessità, come abbiamo visto, dagli anni '70 in poi, sono tornate al centro dell'economia le persone. E le persone non sono oggetti ma sono entità che vivono in un contesto, in una società, in una storia e in una cultura. Ossia 
in un territorio che tutte queste cose porta con sé, nel corso del tempo. Le differenze che emergono, in questo modo di produrre, non sono solo frutto della singola persona ma di un contesto più vasto. Usando la prossimità e la storia come collante le persone ritornate in scena nel contesto post-fordista hanno favorito la trasformazione delle forme organizzative ereditate dal fordismo.

Le vecchie aziende, una volta integrate verticalmente, con tutte le funzioni e le competenze racchiuse nel cerchio proprietario, hanno espulso fuori - con l'outsourcing - una gran parte della produzione svolta in precedenza all'interno, portando fuori parti sempre più estese della propria filiera produttiva. Oggi nelle 4500 medie imprese industriali, che sono l'ossatura del capitalismo industriale italiano, il $79 \%$ valore aggiunto è prodotto da altri, cioè dai fornitori che vendono all'impresa committente materie prime, energia, semilavorati, componenti, lavorazioni conto terzi, conoscenze, consulenze, servizi.

Nel momento in cui una parte importante della filiera che stava dentro il bozzolo della rete fordista è uscita fuori dai confini proprietari, è atterrata nel territorio circostante o in segmenti territoriali collegati, che si sono specializzati in certe funzioni, proprio per riceverla.

La prossimità geografica e professionale (molti dei nuovi micro-imprenditori esterni sono ex dipendenti della grande impresa impegnata nell'outsourcing) è un potente collante, che inizialmente lega le attività in uscita dalla grande organizzazione e poi favorisce la proliferazione locale di nuove imprese e di nuovi specialisti distrettuali. La rete cognitiva che nell'agenda fordista era confinata entro la sfera proprietaria, dagli anni 70 poi si è, infatti, proiettata e stratificata all'esterno, trovando nel territorio il supporto necessario per addensare competenze e specializzazioni richieste dalla filiera.

Anche in questo caso, come si vede, le singole aziende hanno preso iniziative individuali per conto proprio, senza alcuna decisione presa dall'alto: hanno innovato, sono state fondate ex novo, hanno imitato quelle che già c'erano, hanno cercato il rapporto con i committenti e con gli specialisti locali, si sono specializzate in questa o quella competenza richiesta dal distretto. Tutto in funzione delle proprie idee, dei propri capitali, dei rischi assunti personalmente dai singoli.

Ma queste iniziative hanno avuto successo perché hanno messo le vele al vento della storia, facendo surfing sull'onda che fa avanzare il paradigma allora emergente (il capitalismo della flessibilità).

Hanno trovato, prima di tutto, una domanda elevata e insoddisfatta di flessibilità che, in Europa e in America, scaturiva dalla crisi del fordismo, e dagli inciampi provocati dalla rigidità delle sue strutture, nonché dalla lentezza dei suoi processi di adattamento. Trascinato dalla crisi del precedente paradigma, il nascente capitalismo della flessibilità forniva alle aziende che ad esso si affidavano anche risposte efficaci alle loro esigenze: la piattaforma territoriale, in cui ogni singolo imprenditore può trovare tutto quello che gli serve per la sua navigazione individuale (specialisti, buyers, lavoro professionalizzato, fornitori affidabili, conoscenze diffuse nell'“atmosfera" marshalliana del distretto ecc.). 
Di questo rapporto cruciale - tra i successi individuali e l'emergere di un paradigma nuovo che traina le iniziative con esso coerenti - c'è poca memoria. Con una forma singolare di dimenticanza storica e biografica molti degli attuali imprenditori, o i loro eredi, sono convinti di "aver fatto tutto da soli". Rendendo omaggio alla loro indubbia capacità e al loro coraggio o intraprendenza, bisogna anche sapere che c'era un convitato di pietra a fianco del loro lavorare e rischiare: il territorio, che forniva alle singole iniziative l'energia del paradigma emergente. Erano bravi, a vincere il confronto competitivo sui mercati, ma la loro bravura in altri tempi - prima o dopo il ciclo d'oro distrettuale 1970-2000 - non avrebbe avuto lo stesso successo.

Oggi, il surf va fatto sulle nuove onde che accompagnano l'emergere del capitalismo globale della conoscenza, il paradigma destinato a dominare i primi decenni del nostro secolo. Questo significa che le innovazioni non possono più semplicemente appoggiarsi sulle piattaforme territoriali (locali) ma devono sfruttare le differenze tra i tanti e diversi luoghi presenti nell'economia globale. È la relazione tra i territori che conta, e dunque la loro specializzazione reciproca, la loro capacità di lavorare in un circuito efficace di divisione del lavoro in cui ognuno rinuncia a qualcosa per lasciare spazio ad altri, e chiede - su questo - reciprocità agli altri protagonisti della filiera.

\section{Che cosa diventa il territorio?}

Il territorio cambia, ancora una volta, funzione e identità. Nel nuovo paradigma diventa per un verso un sistema che alimenta, al suo interno, la crescita di conoscenza generativa (specifica e non mobile); per un altro verso, il territorio si fa piattaforma, nodo di reti che sono proiettate verso l'esterno e la grande distanza.

In ogni territorio oggi prende forma una specifica combinazione di:

1. attività generative ad alto valore aggiunto (non mobili), in esso localizzate, che forniscono innovazioni, idee motrici, esperienze di frontiera, identità e competenze distintive;

2. risorse connettive (logistiche, comunicative, di garanzia e di presidio delle risorse e competenze critiche nella filiera), che forniscono ai suoi abitanti dei gates di accesso verso il mondo (non mobili);

3. prodotti e soluzioni replicative che vengono messe a punto e modellizzate (in prototipi e esperienze sperimentali) nel territorio di origine, per essere poi trasferite e replicate nel grande circuito globale mantenendo un certo grado di controllo sugli usi.

Non si tratta di un assetto statico, ma dinamico: i prodotti e le soluzioni replicative si trasferiscono altrove, generando grandi moltiplicatori di valore man mano che il loro bacino di uso si estende. Poi, col passare del tempo prodotti e soluzioni replicative si banalizzano e perdono valore. Nel frattempo, tuttavia, una parte più o meno grande, del surplus prodotto dalla filiera generativa/replicativa è stata catturata dalle imprese e dalle persone (o dalle istituzioni) presenti nel 
territorio, e va ad alimentare la crescita del capitale generativo e connettivo ivi localizzato.

Non tutti i territori sono uguali, da questo punto di vista. C'è infatti chi - in questo processo dinamico dove alcune cose entrano e altre escono - riesce a mantenere in equilibrio o in crescita il movimento delle cose. E c'è chi, invece, ne viene travolto perché perde attività replicative senza riuscire a catturare una quota del surplus dovuto al loro ri-uso, non avendo così mezzi per finanziare l'accumulazione generativa e connettiva nel sistema locale di appartenenza. Ci sono poi territori che respingono, per vari motivi, le localizzazioni generative e connettive provenienti dall'esterno, non facendo abbastanza per richiamarle e allontanandole volontariamente in un atteggiamento che non promette niente di buono per il futuro. Ma ci sono anche territori che fanno il contrario, varando politiche attive di attrazione (incentivi, regole, impegni ecc.) che si aggiungono ai fattori localizzativi favorevoli esistenti in loco.

Dunque, i percorsi possibili sono tanti e diversi tra loro. Possiamo però dire una cosa: il territorio che diventa snodo o ancoraggio specializzato di filiere transterritoriali è destinato a cambiare la propria identità storica, in misura più o meno grande, per venire incontro alle esigenze funzionali e alle opportunità offerte dall'interscambio con la filiera e con gli altri territori che essa mette in collegamento. In questa trasformazione che cambia, giorno per giorno, modelli di business e fattori distintivi, l'identità del territorio cessa di essere data (dalla natura o dalla storia) e diventa invece, almeno in parte, oggetto di scelta, da parte dei soggetti che nel territorio vivono e operano.

Non sono milanese o torinese perché sono nato a Milano o Torino (identità data, dovuta alle origini), ma sono milanese o torinese perché scelgo di essere parte di uno di questi sistemi territoriali, legandomi alle sue caratteristiche per me positive; e scelgo di farne parte perché, essendo il sistema territoriale in evoluzione, posso sfruttare a mio vantaggio l'energia che muove di questa trasformazione macro, contando anche di cambiare - in parte - la qualità del sistema territoriale a cui mi lego.

\section{II nuovo ruolo delle economie di prossimità}

Il terzo nucleo di discussione emerso dal convegno riguarda le economie di prossimità. I sistemi locali lavorano, essenzialmente, sui vantaggi/svantaggi relativi alla vicinanza fisica, o geografica. Che non sono soltanto relativi ai costi di trasporto (a distanza ravvicinata) ma riguardano i costi e benefici del clustering delle conoscenze, delle professionalità, delle relazioni che si materializza in un certo contesto territoriale.

La dimensione globale è quella in cui sono iscritti tutti i processi in cui la barriera della distanza viene ridotta (grazie ai trasporti di persone e di beni materiali) o tolta del tutto (grazie alle ICT che spostano a distanza, in tempo reale, informazioni e conoscenze replicative a costo zero). Ma non tutte le funzioni 
presenti nelle filiere globali hanno queste caratteristiche. Anche se possiamo codificare le cose e mandare a distanza le loro rappresentazioni, resta vero che molte delle conoscenze utilizzate nella vita o nel lavoro sono troppo complesse (varie, variabili e indeterminate) per essere affidate a questi circuiti globali. Essendo legate alle persone e ai contesti, esse sono infatti comunicabili di persona, de visu, insieme. Per effetto di questo legame, esse rimangono nei sistemi locali o al massimo si distribuiscono nello spazio metropolitano, che comprende un milione, due milioni di persone (come accade ai servizi rari, che necessitano di un grande bacino di potenziale domanda).

Nel territorio si realizza dunque l'accoppiamento tra le attività generative e connettive della filiera (rete cognitiva, rete relazionale) e le economie di prossimità, per tutto ciò che nasce o è decentrato fuori dei confini proprietari della singola impresa. Per essere fatto "nel territorio".

Le onde nuove che alimentano questo rapporto problematico tra attività generative locali e attività replicative di filiera, si chiamano oggi globalizzazione, e quindi sfruttamento delle differenze; e si chiamano smaterializzazione, quindi creazione dei significati.

\section{Anche la nostra disciplina ha scandito i tempi della successione dei paradigmi}

La dottrina comincia a riconoscere non solo questa nuova fisionomia multiterritoriale della produzione di valore, ma anche il fatto che essa arriva dopo una serie di passaggi, appoggiati ai diversi paradigmi che hanno dominato la scena passata. Non è qui il caso di passare in rassegna le diverse tappe di questo percorso. Possiamo tuttavia seguire la successione di modelli collegati al succedersi dei paradigmi produttivi, guardando un esempio per tutti: le tappe, molto istruttive, che scandiscono il tempo nella produzione scientifica di Michael Porter, un esempio di eccellenza disciplinare, a tutti noto.

Se seguiamo anche a volo d'uccello la sua lunga carriera di studioso di management, possiamo scoprire, abbastanza nettamente, tutte le fasi di cui abbiamo parlato.

Prima, quando Porter inizia ad essere un autore di riferimento la sua proposta concettuale chiave è quella del vantaggio competitivo che differenzia la singola impresa nella competizione di mercato (Porter, 1986). Col senno di poi, non facciamo fatica a vedere nella rappresentazione di questa impresa - dotata di una forte differenziazione identitaria e priva di un contesto territoriale attivo - la tipica impresa fordista, che giunge a maturità nella seconda metà del novecento.

L'analisi del vantaggio competitivo in sistemi complessi come quelli fordisti che comprendono un numero davvero rilevante di funzioni e specializzazioni concatenate in sequenza o diversificate, richiede uno strumento di analisi che dia conto degli specifici punti di forza e di debolezza (competitiva) di ciascuna funzione e specializzazione interna. Porter propone l'idea della "catena del valore": la 
compattezza della grande organizzazione tende, nella value chain, a frammentarsi, man mano che il processo di generazione interna del valore si scompone in fasi e in nodi, che il management può separare, ricombinare, misurare e guidare separatamente. Il passo successivo è breve, e scontato: con l'outsourcing degli anni ottanta alcune delle funzioni sono uscite dai confini proprietari e, di conseguenza, la catena (interna) del valore è diventata una costellazione, ossia un sistema pluriimprenditoriale che crea valore mettendo insieme il contributo attivo di molte soggettività, compresa quella dei consumatori finali (Normann e Ramirez, 1993).

Contemporaneamente, la fuoriuscita del sistema del valore dai confini proprietari aziendali ha investito il territorio e lo ha specializzato in soluzioni connettive e organizzative differenti in ogni paese. Le costellazioni del valore si appoggiano dunque a contesti territoriali diversi, creando sinergie e simbiosi che legano i territori alle imprese in essi localizzate e viceversa. La globalizzazione degli anni ottanta e novanta fa emergere una pluralità di modelli nazionali (quello giapponese e quello italiano, in particolare), che si affiancano al modello una volta leader, quello americano. Dunque - portando il discorso sui modelli nazionali - si comincia a parlare di catene del valore differenziate da paese a paese: ciascuna di esse presidia il vantaggio competitivo della Nazione a cui è ancorata, dando accesso ai fattori distintivi che differenziano i vari contesti nazionali (Porter, 1989).

Di recente, guardando nuovamente alla fisionomia del vantaggio competitivo e della catena del valore che lo crea, Michael Porter fa, insieme a Mark Kramer, un ulteriore passo e - avendo in mente la distribuzione delle funzioni nelle filiere globali - afferma che dobbiamo familiarizzarci con un nuovo modo di produrre, in cui il valore risultante è condiviso (shared value) tra i molti partecipanti che contribuiscono alla filiera: fornitori, committenti, distributori e anche consumatori finali. L'impresa di oggi, grande o piccola che sia, co-produce con altri il valore offerto al consumatore, usando piattaforme territoriali diverse e complementari, congiunte dai flussi di filiera (Porter e Kramer, 2011). Una concezione che rimanda a processi non solo di interdipendenza "responsabile" nelle filiere globali, ma anche di co-innovazione e di gestione condivisa delle regole e degli sviluppi delle relazioni nei sistemi di shared value.

\section{I tre sistemi di relazione che si sovrappongono nel territorio}

La direzione di marcia verso cui va l'economia di impresa, in rapporto al territorio, sembra chiara: il processo del valore eccede ormai di gran lunga il circuito proprietario della singola impresa, e si allarga alla filiera, alla rete cognitiva, alle economie di prossimità del circuiti locali. Globalizzazione e smaterializzazione lo stanno portando lontano, imponendo fasi che compongono le filiere di disaccoppiarsi guadagnando una distanza e una differenziazione tra loro. La filiera che diventa globale pone al singolo territorio domande inequivocabili. Prima di tutto, dove collocarsi nella filiera: quali fasi della filiera tenere? Quali fasi cedere ad 
altri? Quali delle proprie qualità valorizzare e quali mettere, invece, in seconda fila (perché meno utili alla collocazione funzionale voluta)?

La nuova identità di ciascun territorio dipenderà dalla fusione - differente da caso a caso - di tre sistemi di relazione:

- la filiera, tendenzialmente globale, che mette insieme le operazioni necessarie per la creazione di valore in funzione di un certo prodotto (vendibile), da collocare sul mercato;

- la rete cognitiva, che tiene insieme le fonti cognitive potenzialmente utili alle decisioni da prendere e alle operazioni da fare. La rete cognitiva non sarà uguale alla filiera, perché riguarda le conoscenze potenzialmente utili (che eccedono sempre di molto quelle usate nelle operazioni attuali)

- il cluster, che addensa la rete di prossimità, centrata per un verso sui vantaggi logistici e comunicativi della vicinanza fisica, e per un altro sulla scarsa mobilità delle conoscenze generative e delle risorse connettive, che tendono a cumularsi in un luogo.

In passato, filiera, rete cognitiva e cluster di prossimità tendevano a sovrapporsi e ad essere, alla fine, la stessa cosa, sinteticamente chiamata "territorio".

Oggi questi elementi si stanno disaccoppiando, perché le filiere e le reti cognitive si allungano verso la dimensione globale, separandosi - per tutta una serie di funzioni - dal cluster di prossimità.

Molti dei nostri territori, in Italia, da qualche tempo hanno cominciato ad alimentare al proprio interno il conflitto latente - e in certi casi manifesto - tra queste istanze che, certo, possono svolgere un ruolo complementare, ma che - in mancanza di una sintesi unitaria - si avviano e crescono, appunto, in modo conflittuale.

Il territorio si trova infatti stretto tra tre dinamiche non sempre compatibili e ben sincronizzate. Esso deve infatti: diventare uno snodo di filiere globali che hanno logiche e interessi trans-territoriali; organizzare e muoversi in una rete cognitiva anch'essa amplissima, ma varia e fluttuante, per imparare a fare cose nuove, meglio e prima di altri; investire nella accumulazione di un capitale di conoscenze generative e di risorse connettive locali, destinate ad essere valorizzate nella filiera e nelle reti cognitive globali.

Non è facile mettere d'accordo i tempi e le direzioni di queste tre istanze. Ma il territorio oggi è questo, e non può sottrarsi al conflitto tra le sue tre traiettorie evolutive perché è da esse che riceve l'energia necessaria a governare la transizione in corso. Il dibattito che il convegno di Sinergie ha dedicato a questi risvolti dell'azione imprenditoriale sul territorio è stato ricco di spunti e anche di visioni contrastanti. Ma penso che non sia utile, né metodologicamente corretto, semplificare a forza una realtà che, sommando vecchio e nuovo, paradigmi in declino e paradigma emergente, assume tante facce quante sono le possibili vie da prendere.

Aggiungiamo alla collezione di immagini che il convegno ci ha lasciato, un'ultima rappresentazione: quella di una schiera di imprese che, per innovare, si attrezza per cavalcare le onde e le contraddizioni emergenti, nel mare in tempesta. 
Le onde porteranno in alto i nostri surfisti, e molti di loro voleranno in avanti, bruciando le tappe. Il resto, a Dio piacendo, seguirà.

\section{Bibliografia}

DI BERNARDO B., RULLANI E. (1985), Transizione tecnologica e strategie evolutive: l'impresa industriale verso l'automazione, Padova, Cedam.

DI BERNARDO B., RULLANI E. (1990), Il management e le macchine. Teoria evolutiva dell'impresa, Il Mulino, Bologna.

NORMANN R., RAMIREZ R. (1993), "From value chain to value constellation: designing interactive strategy", Harvard Business Review, luglio-agosto, pp. 65-77.

PLECHERO M. (2012), The changing geography of innovation. Chinese and Indian regions and the global flows of innovation, Lund University Centre for Innovation, Reseearch and Competence in the Learning Economy (CIRCLE).

PORTER M.E. (1986), The Competitive Advantage. Creating and Sustaining Superior Performance, The Free Press, New York.

PORTER M.E. (1989), The Competitive Advantage of Nations, The Free Press, New York.

PORTER M.E., KRAMER M.R. (2011), "Creating shared value. How to reinvent capitalism and unleash a wave of innovation and growth", Harvard Business Review, Januaryfebruary, pp. pp. 62-77.

RULLANI E. (2010), "La conoscenza e l'organizzazione. Promesse e minacce dei sistemi incompiuti", Rassegna Italiana di Sociologia, anno 5lesimo, n. 2, aprile-giugno, pp. 303-324

SIMON H.A. (1982), "Models of Bounded Rationality", Behavioural Economics and Business Organization, vol. 2, The MIT Press, Cambridge (MA). 
